\title{
BIBLIOGRAPHIE DER ARBEITERBEWEGUNG HEUTE UND MORGEN*
}

Welchen Platz nimmt die Bibliographie der Arbeiterbewegung und des Sozialismus heute ein und wie füllt sie ihn aus? Der beträchtliche Aufschwung, den in den letzten 15 Jahren die Historiographie auf diesem Gebiet erfahren hat, fand seinen Ausdruck auch in erheblich vermehrter bibliographischer Tätigkeit. Die Zahl der in den letzten 10 bis 15 Jahren veröffentlichten Bibliographien ist bedeutend höher als die der im vorhergegangenen halben Jahrhundert gedruckten. ${ }^{1}$ Der Historiker der Arbeiterbewegung, wie übrigens auch manche seiner Kollegen anderer Spezialgebiete, benutzt häufig die ihm von den Hilfswissenschaften zur Verfügung gestellten Arbeitsinstrumente, insbesondere Bibliographien, ohne die ihrer Herstellung eigenen Bedingun-

* Dieser Text hat (ohne Anmerkungen) der zweiten Internationalen wissenschaftlichen Tagung zur Geschichte der Arbeiterbewegung vorgelegen, die vom 8. bis 10 . September 1966 stattfand.

1 Für ein Spezialgebiet ist diese Entwicklung weiter unten (S. 28, Anm. 1) anschaulich dargestellt. An ihrem Anfang stehen die klassischen Werke von Josef Stammhammer, Bibliographie des Socialismus und Communismus, Jena 1893-1909, 3 Bde, IV-303 \& IV-403-[1] \& [4]-473-[1] S. (fotomechanische Neuausgabe bei Otto Zeller, Aalen, 1964) und von Max Nettlau, Bibliographie de l'Anarchie, Brüssel/Paris 1897, XI-[1]-294 S. Von den vor 1950 erschienenen wichtigeren Bibliographien seien noch erwähnt: Johann Sassenbach, Verzeichnis der in deutscher Sprache vorhandenen Gewerkschaftsliteratur, Berlin, Generalkommission der deutschen Gewerkschaften, Heft I [4. erw. Auflage] \& II [Nachtrag], $1910 \&$ \& 1912, 32 \& X-[27] S.; Ju. K[amenev], Social' demokratiČeskaja izdanija. Ukazatel' social'demokraticeskoj literatury na russkom jazyké 1883-1905 gg., Paris 1913, 56-[2] S.; Ernst Drahn, Bibliographie des wissenschaftlichen Sozialismus 1914-1922, Berlin, Russ. Volkskommiss. f. Bildungswesen, 1923, VIII-159-[1] S. (mit einer stoffreichen Einleitung ,Zur Bücherkunde des deutschen Sozialismus", S. 1-17); Michel Ralea, Révolution et socialisme. Essai de bibliographie, Paris, P.U.F., 1923. Vgl. S. 9, Anm. 2 und S. 20, Anm. 3 für Arbeiten von Czóbel und Kormanowa. In den Jahren von 1920 bis 1930 sind in der Sowjetunion zahlreiche bibliographische Arbeiten zur russischen und zur internationalen Arbeiterbewegung erschienen. Eine kritische Bibliographie dieser Arbeiten, die im Westen so gut wie unbekannt sind, würde sehr nützlich sein. 
gen und Probleme näher zu kennen. Dies mangelnde Verständnis ist der Forschung im allgemeinen abträglich und führt, wie wir weiterhin sehen werden, zu Kraftvergeudung. In einzelnen Fällen kann man sich des Eindrucks nicht erwehren, die Geschichtsschreibung sei der Bibliographie vorausgeeilt, die unumgängliche bibliographische Vorbereitung der Arbeit durch den Historiker sei unzulänglich gewesen oder jedenfalls nicht von dem dazu berufenen Spezialisten durchgeführt worden. Die eigentliche Ursache solcher Erscheinungen ist weniger in Uebereilung des Historikers oder im Zurückbleiben des Bibliographen zu suchen, sondern vielmehr in mangelnder Koordination beider Tätigkeiten und infolgedessen ungenügend formulierten Ansprüchen an die Qualität der von der Hilfswissenschaft geforderten Arbeit. Auf die Frage der Koordination werden wir noch zurückkommen. Wenden wir uns zuerst, ohne jegliche polemische Absicht, einigen Fragen zu, die uns für den Bibliographen wesentlich zu sein scheinen. 1. Die Isolierung der Bibliographen führt zu einer bedeutenden Kraftverschwendung. Gar nicht selten unternehmen zwei oder mehr Forscher ungewusst und unabhängig voneinander eine gleiche Arbeit. So hat kürzlich ein Kölner Forscher eine Stirner-Bibliographie beendet, ${ }^{1}$ während seit langen Jahren ein Mailänder Kollege ebenfalls an einer Stirner-Bibliographie arbeitete, die jetzt wahrscheinlich unveröffentlicht bleiben wird. ${ }^{2}$ Anders als im Falle der Biographie schliessen bei der Bibliographie Materie und Form Wiederholungen praktisch aus. Der Gegenstand einer Biographie erlaubt verschiedenen Autoren unterschiedliche Interpretationen und Darstellungen, die nebeneinander bestehen und sich sogar ergänzen können. Die Bibliographie erlaubt keine Interpretation und stellt einmalige Fakten fest, die für jeden Forscher unveränderlich dieselben bleiben und die er bestenfalls übersichtlicher als ein anderer gruppieren und eventuell annotieren kann.

Verlust von bereits vorhandenen Forschungsergebnissen tritt häufig dadurch ein, dass dem Bibliographen seine eigene Untersuchung interessierende Nebenresultate der Arbeiten anderer Forscher unbekannt bleiben, weil sie in der Veröffentlichung der Hauptresultate keinen Platz gefunden haben. Häufig bleiben Teiluntersuchungen un-

1 Hans-G. Helms, Die Ideologie der anonymen Gesellschaft, Köln 1966, S. 507-600: „Max Stirner Bibliographie”.

${ }^{2}$ Das Feltrinelli-Institut zeigte in seinen Annali 1958, Jg. I, S. 436 an, sein Mitarbeiter Aldo Zanardo bereite unter dem Titel La Sinistra Hegeliana (18351849) Bibliographien folgender Verfasser vor: Bruno und Edgar Bauer, Strauss, Feuerbach, Ruge und Stirner. Inzwischen erschien in der Rivista Critica di Storia della Filosofia, Florenz 1965, „Bruno Bauer hegeliano e giovane hegeliano” von Zanardo (57 S.). 
zugänglich, weil sie entweder nur als nicht nachgewiesenes Manuskript vorliegen oder in geringer Auflage polykopiert nur dem internen Gebrauch bestimmter Institute dienen. Wir sehen hier davon $a b$, Beispiele namhaft zu machen, die den meisten Forschern aus eigener bitterer Erfahrung hinlänglich bekannt sind.

Schliesslich muss die oftmals ungenügende Unterstützung bibliographischer Arbeiten seitens der grossen Institute, und mitunter auch der Fachkollegen, erwähnt werden. Die von den meisten Instituten geführte Geheimhaltepolitik ist sattsam bekannt. Aber auch eine gewisse Unbekümmertheit ist mitunter festzustellen. So ist es zum Beispiel kürzlich vorgekommen, dass in einem Institut, das seine reichen Dokument-Sammlungen einer bestimmten Organisation inventarisierte, die damit beschäftigten Mitarbeiter Namen und Adresse und sogar das Manuskript eines Forschers kannten, der eine allgemeine Bibliographie derselben Organisation fertiggestellt hatte. Sie vergassen aber, ihm ein Exemplar ihres polykopierten Repertoriums zu schicken, das ihm erlaubt haben würde, einige Ergänzungen nachträglich in seine allgemeine Bibliographie aufzunehmen. Die Leidtragenden sind natürlich die künftigen Benutzer der Bibliographie, denen die betreffenden Daten vorenthalten bleiben.

2. Das Fehlen genau definierter Methoden und Kriterien unserer Hilfswissenschaft, zumindest die Uneinheitlichkeit der vorhandenen, sind gewissermassen ihre Achillesferse. Sie wird nur zu deutlich sichtbar, wenn man die drei kürzlich erschienenen Repertorien zur Hand nimmt, welche die gedruckten Quellen der drei grossen internationalen politischen Organisationen der sozialistischen Bewegung zum Gegenstand haben, die Erste, Zweite und Dritte Internationale. Bei allen Besonderheiten der Struktur und der Entwicklung dieser drei Organisationen sind die zu erfassenden Quellen in allen drei Fällen der Natur nach identisch, und man dürfte demnach drei Repertorien gleichen Typus erwarten, deren Zusammensteller gleiche Methoden und Kriterien angewendet hätten. Nichts ist jedoch weniger wahr. Wir haben hier nicht über den Wert oder Unwert der einen oder anderen der drei Arbeiten zu urteilen oder diese oder jene der angewandten Methoden und Kriterien zu empfehlen, werden aber auf einige Fragen zurückzukommen haben, die sich im Zusammenhang mit den drei Arbeiten ergeben.

In der Sammlung Mouvements ouvriers et socialistes (Chronologie et bibliographie), einem wahren Pionier-Werk, sind seit 1950 sechs Bände veröffentlicht worden, die jeweils einem einzigen Lande oder einer Gruppe von Ländern gewidmet sind und die insgesamt ein sehr buntscheckiges Bild ergeben: ihre einzige Gemeinsamkeit besteht im Titel, denn jeder einzelne Verfasser ist von seiner eigenen Auffassung ausge- 
gangen und hat seine eigene Methode und seine besonderen Kriterien angewandt. Einige Bände begnügen sich in der Hauptsache mit einer Chronologie der Arbeiterbewegung, der hier und da einige Buchtitel zur Illustration beigegeben sind. ${ }^{1}$ Andere reproduzieren die Kataloge von mehr oder weniger zahlreichen Bibliotheken, ${ }^{2}$ und die Verfasser eines Bandes haben sich offenbar darauf beschränkt, dem Drucker den Inhalt ihres Zettelkastens zu übergeben. ${ }^{3}$ Es ist jedem Autor überlassen geblieben, den Ausgangspunkt der Chronologie zu bestimmen, und einer hat diese Freiheit benutzt, um bis auf Kolumbus und die Entdeckung Amerikas zurückzugreifen. ${ }^{4}$

Vor etwa zwei Jahren ist in Warschau die Probenummer des Bibliographical Bulletin of Current Marxology erschienen, eines vor allem für die Zukunft wichtigen Unternehmens. ${ }^{5}$ Die Redakteure definieren in der Einleitung ihr Editionsprinzip dahingehend, dass Schriften sowohl von „Anhängern als Kritikern” aufgenommen wurden, soweit sie „einen deutlichen und engen Zusammenhang" mit dem Thema haben. Doch so, wie die Redakteure den Ausdruck „Marxologie” anwenden, umfasst er ungefähr alles, und er bedeutet nichts Genaues, denn man findet in ihrer Bibliographie Seite an Seite soziologische Sammelwerke und Spezialstudien über Einzelaspekte der Arbeiterbewegung.

3. Bis jetzt haben wir uns hauptsächlich zum Dolmetscher von Beschwerden gemacht, welche die Historiker, und häufig zu Recht, gegen bibliographische Arbeiten vorbringen. Betrachten wir nunmehr die Kehrseite der Medaille. Die Arbeit des Bibliographen ist, wie bekannt, meistens langwierig und mitunter langweilig, und das Resultat jahrelanger Arbeit findet oft nicht die erhoffte Anerkennung. $\mathrm{Zu} \mathrm{dem}$ Mangel an Verständnis für diese undankbare Arbeit tritt häufig eine gewisse Geringschätzung des Bibliographen, die einen der grossen Historiker unserer Zeit zu der folgenden Zurechtweisung veranlasst hat:

„Eine der schwierigsten Aufgaben des Historikers besteht in dem Zusammentragen der Dokumente, die er für seine Arbeit zu

1 Alfonso Leonetti, L'Italie (Des origines à 1922), Paris, Ed. ouvr., 1952, 198[2] $\mathrm{S}$.

2 Eugène Zaleski, La Russie 1725-1907, 1908-1917, Paris, Ed. ouvr., 1956, 2 Bde, $462-[1] \& 489-[3] \mathrm{S}$.

3 Edouard Dolleans - Michel Crozier, Angleterre, France, Allemagne, Etats-Unis (1750-1978), Paris, Ed. ouvr., 1950, XVI-381-[2] S. Bedeutend vollständiger dagegen ist Renée Lamberet, L'Espagne (7750-1936), Paris, Ed. ouvr., 1953, 204-[3] S.

4 Carlos Rama, L'Amérique Latine (1492-1936), Paris, Ed. ouvr., 1959, 222-[2] S.

5 Bibliographical Bulletin of Current Marxology. Trial Issue, Warschau 1965, 176 S. Polykopie. Führt 745 Titel von im Jahre 1962 erschienenen Büchern und Zeitschriften-Artikeln an, die nach Ländern geordnet sind. Die meisten Titel sind kurz erläutert, häufig mit einer Zusammenfassung des Inhalts. 
benötigen glaubt. Er würde sie kaum bewältigen können ohne die Hilfe verschiedener Wegweiser: Archiv-Inventare, Bibliotheksund Museums-Kataloge, bibliographische Repertorien aller Art. Man hört mitunter Schulmeisterlein auf hohem Ross sich über die viele Zeit wundern, die einige Gelehrte dem Zusammenstellen derartiger Werke opfern und die alle wissenschaftlichen Arbeiter aufwenden, um deren Vorhandensein und Handhabung zu erlernen. Als ob nicht schliesslich eben die vielen, auf diese wenn auch nicht gänzlich reizlose, dann doch jeglichen Glanzes entbehrende Beschäftigung verwendeten Stunden uns die abscheulichste Kraftvergeudung ersparten!"1

Aus der fehlenden Koordination von Historikern und Bibliographen, der Isolierung der letzteren, der uneinheitlichen Auffassungen über Platz, Rolle und Bedeutung der Bibliographie, dieser verkannten Hilfswissenschaft, ergeben sich Folgen, für die Abhilfe gefunden werden muss. Zum Beispiel sehen die Universitätsprogramme nur selten eine Einführung in die Bibliographie vor. Bei der immer noch vorherrschenden engen, traditionalistischen Auffassung des BibliographenBerufes wird die Nachwuchsfrage immer schwieriger zu lösen sein. Zitieren wir noch einmal Marc Bloch: „Gewiss, das Werkzeug schafft nicht die Wissenschaft. Aber eine Gesellschaft, welche die Wissenschaften zu achten vorgibt, sollte sich nicht achtlos gegenüber deren Werkzeugen verhalten."

Der Nachwuchs sollte nicht aus uninteressierten Zusammenstellern bestehen, sondern aus Bibliographie-Forschern, die als Historiker und Dokumentalisten qualifiziert sind und über streng definierte Methoden ihrer Hilfswissenschaft verfügen. Um dies zu erreichen, müssen zahlreiche Fragen geklärt und Definitionen ausgearbeitet werden. Wir müssen uns hier darauf beschränken, einige Punkte zu nennen und vielleicht zum Nachdenken anzuregen.

\section{II}

Die jeweilige Beschaffenheit der Probleme, die Menge und Unterschiedlichkeit der zu erfassenden Dokumente und die unterschiedlichen Bedürfnisse der Geschichtsforschung erfordern ihnen entsprechende Arbeitsinstrumente, d.h. Bibliographien verschiedener Art, die ihrerseits spezifische Probleme aufgeben und entsprechende Kompetenzen erfordern. Dies setzt bei den Bibliographen eine vielseitige Ausbildung voraus, die sie befähigt, den jeweiligen Ansprüchen gerecht zu werden. 1. Die einfachste und gleichzeitig von der Forschung am häufigsten

${ }^{1}$ Marc Bloch, Apologie pour l'Histoire ou Métier d'historien, Paris, Colin, 1949, p. 29. 
verlangte Art ist die laufende Bibliographie, deren Redaktionstechnik weitgehend der einfachen Dokumentation entspricht. ${ }^{1}$ Die Entwicklung neuer Dokumentations-Techniken durch Benutzung von Maschinen wird in naher Zukunft eine schnellere und vollständigere Information erlauben, sobald der Bibliograph die neue Technik zu beherrschen versteht. Bis jetzt sind die Leistungen auf diesem Gebiete sehr ungleichmässig. Die bisher (meistens in Fachzeitschriften) erschienenen laufenden Bibliographien sind auf verschiedene Art unvollständig: sie sind entweder beschränkt auf die von der Redaktion empfangene Literatur, oder auf eine geographisch, oder auf eine ideologisch bestimmte Auswahl. ${ }^{2}$ Unter diesen Umständen hängt der Forscher-Benutzer mehr vom glücklichen Zufall ab, und er kann sich nicht auf eine zuverlässige, systematische Information stützen. Auch hier erscheint baldige Koordination geboten, da sonst in naher Zukunft die inflatorisch wachsende $Z$ ahl der Veröffentlichungen jegliche bibliographische Untersuchung in Frage stellen wird, die auch nur eine annähernde Vollständigkeit anstrebt.

2. Die retrospektive Bibliographie verschafft einen Ueberblick über die gesamte Geschichtsliteratur eines bestimmten Gegenstandes. In dem Masse, wie diese Art der Bibliographie mehr als eine einfache Titelliste sein und ein brauchbares, leicht hantierbares und nützliches Arbeitsinstrument werden soll, muss der Bibliograph zugleich Historiker und Philologe sein. Denn die Literaturübersicht, welche er liefert, gewissermassen eine Leistungs-Bilanz der Geschichtsschreibung, sollte auf der Analyse der erfassten Titel fussen, sie sollte implizit und explizit eine kritische Bibliographie sein. Bei seinem Streben nach Vollständigkeit bringt der Verfasser unvermeidlich Texte von sehr unterschiedlichem Wert und Charakter zusammen, neben gelehrten Untersuchungen auch Gelegenheitsschriften und Propagandabroschüren. Die Kritik kann

1 Als Beispiel einer solchen Dokumentation sei die Internationale Bibliographie marxistischer Zeitschriftenliteratur (Berlin, Dietz, 1951-1962, 11 Bde von durchschnittlich $100 \mathrm{~S}$.) genannt, in der Artikel von Juli 1950 bis Dezember 1959 nachgewiesen werden. Die seit Januar 1960 erschienenen Artikel werden erfasst in der gleichnamigen Bibliographie, die seit 1960 regelmässig erscheint in Beiträge zur Geschichte der deutschen Arbeiterbewegung.

2 Auf die Literatur ihrer Länder beschränken sich beispielsweise die laufenden Bibliographien im Bulletin of the Society for the Study of Labour History (London 1960-1966, NN. 1, 3, 4, 5, 7, 10, 12) und im Bulletin (Canberra 1962, Nr. 1) der gleichnamigen australischen Gesellschaft. Auf Verfasser einer bestimmten Ideologie beschränkt sich die vom Berliner IML zusammengestellte in der vorhergehenden Anmerkung angeführte Bibliographie. Der vom selben Institut bearbeitete Dokumentations-Dienst. Geschichte der deutschen Avbeiterbewegung macht diese Einschränkung nicht; diese Bibliographie erscheint in Form von Karteikarten (etwa 300 im Laufe eines Jahres) mit kurzer Inhaltsangabe und kritischer Wertung. 
implizit erfolgen durch Entscheidungen, welche eine Hierarchie der Werte herstellen, durch Einteilung in Gruppen, oder durch drucktechnische Unterscheidungen, oder Verweisung in einen Anhang. Der Bibliograph sollte ausserdem explizit kritisieren durch Werturteile, z.B. in einer Einleitung oder in erklärenden Noten. Derartige kritische Bibliographien fehlen heute noch. Es gibt jedoch schon mehrere Arbeiten, die annähernd diesem Typus entsprechen und das Ausarbeiten von verbesserten und vollständigeren Bibliographien erleichtern werden. ${ }^{1}$

3. Das Repertorium gedruckter Quellen gehört zu den umfassendsten und vielseitigsten Varianten der Bibliographie. Trotz seiner bibliographischen Form gehört es teilweise eher der Quellenkunde an, und zwar durch die ihm zufallende Rolle und vor allem wegen des Quellenstandes, den die Geschichte der Arbeiterbewegung und des Sozialismus vorfindet.

Seitdem die Bewegung ihre Anfangs- und Vorbereitungsphase verlassen hat, d.h. seit dem Auftreten der Ersten Internationale, sind ihre Hauptquellen, die von ihr ausgegangenen authentischen Dokumente, Ausdruck und Zeugen ihrer Tätigkeit, mit wenigen Ausnahmen schon zur Zeit dieser Tätigkeit selbst gedruckt worden. Wir besitzen zahlreiche Adressen, Rundschreiben, Kongressprotokolle und sogar Sitzungsberichte führender Organe auf verschiedenen Ebenen. Von den Protokollen der internationalen, nationalen und sogar der lokalen Kongresse hat Ernest Labrousse gesagt, welche „bedeutende und bequeme Quelle” sie bilden „für Autoren, die es derart eilig haben, dass sie mitunter aus ihnen ihre einzige Quelle machen". Labrousse hat aber auch hervorgehoben, dass die Protokolle so "vielfach verwertbar sind, dass sie sowohl soziologischen Untersuchungen als bildhaften Darstellungen" dienen. ${ }^{2}$ Die Wichtigkeit dieser gedruckten Quellen ist umso grösser, als die meisten Archive der Organisationen selbst verschwunden sind oder erhaltene sich in sehr schlechtem $\mathrm{Zu}$ stande befinden, wie z.B. die des spanischen Föderalrats der Ersten

1 Dies gilt besonders für die in der folgenden Anmerkung und S. 20, Anm. 3 genannten Bibliographien von Haupt und Hubert. Die ersten Elemente einer Kommune-Bibliographie findet man in dem Inventar der Kommune-Sammlung des Feltrinelli-Instituts, Giuseppe Del Bo, La Comune di Parigi, Mailand 1957, VI-142-[1] S.; als Ergänzung hierzu veröffentlichten Jacques Rougerie und Georges Haupt „Bibliographie de la Commune de 1871. Travaux parus de 1940 à 1961" (500 Titel), in: Mouvement Social, Paris 1961 und 1962, Nr. 37, S. 70-92 \& Nr. 38, 51-85.

2 Ernest Labrousse, S. 12 im Vorwort zu Georges Haupt, La Deuxième Internationale, 1889-1914. Etude critique des sources, essai bibliographique, Paris-Haag, Mouton, 1964, $393 \mathrm{~S}$. 
Internationale, die zum grossen Teil unlesbar geworden sind ${ }^{1}$. Uebrigens ist in sehr vielen Fällen der zwischen gedruckter Quelle und Manuskript zu machende Unterschied ein rein formaler. Kautsky hat schon 1893 darüber geklagt, dass nicht nur „die öffentlichen Bibliotheken achtlos an der Literatur [der Arbeiterbewegung] vorübergingen", sondern auch die Bewegung selbst ihre eigenen Dokumente "nicht zu sammeln suchte”. ${ }^{2}$ Mehr als siebzig Jahre nach Kautsky's Klage sind eine grosse Anzahl auch der gedruckten Dokumente bibliophile Seltenheiten geworden (die immer zahlreicher werdenden photographischen Neudrucke bezeugen es), so z.B. die gedruckten und mitunter auch nur polykopierten Dokumente, die zu rein organisationsinternem Gebrauch bestimmt waren, wie die Berichte des Sekretariats der Zweiten Internationale. Ihre Auflage betrug nur 50 Exemplare, und es gibt heute keine einzige vollständige Sammlung mehr. Hierher gehören vor allem auch die illegale Presse seit eh und je und die meistens handgeschriebenen Informationsblätter, welche von Widerstandsgruppen während des letzten Krieges verbreitet wurden. ${ }^{3}$ Tatsächlich haben heute bereits viele Archivare diese Sachlage erkannt und in bestimmten Fällen auf die Unterscheidung von handschriftlichen und gedruckten Dokumenten verzichtet, die sie zusammen aufführen.

Aber die Wichtigkeit eines Dokumentes beruht so wenig auf seiner Seltenheit wie auf seinem Umfange. Sie entspricht dem RepräsentativWert und der Tragweite; der Seltenheitswert fällt erst dann ins Gewicht, wenn wenig oder keine gleichartigen Dokumente vorhanden sind. Bei den Dokumenten der Arbeiterbewegung müssen weiterhin die ihr ganz eigenen Formen des Organisationslebens und ihrer Veröffentlichungen in Betracht gezogen werden. Beide Erwägungen gelten für die Kongress-Dokumente. Hier ist es wiederum Labrousse, der die der Arbeiterbewegung eigentümliche Besonderheit der Quellenlage und ihre Bedeutung treffend gekennzeichnet hat: „Diese Literatur übertrifft durch ihre Periodizität, ihren Repräsentativ-Charakter und ihre Vielumfassendheit bei weitem alles, was uns seit einem Jahr-

1 Die in der Biblioteca Arús in Barcelona bewahrten Protokolle von 134 Sitzungen des Föderalrates (1870-1874) und Kopien von rund 2500 Briefen und Zirkularen wurden 1928-29 zuletzt von Nettlau benutzt, der bereits über ihre schlechte Erhaltung klagte. Die Archive sind neuerdings wieder bedingt zugänglich gemacht worden, und sie sollen von dem spanischen Historiker $\mathrm{C}$. Seco zur Veröffentlichung bearbeitet werden.

2 Karl Kautsky, ,J. Stammhammer, Bibliographie des Sozialismus und Kommunismus", in : Neue Zeit, September 1893, Jg. XII, Bd 1, S. 25-26.

- Methodologisch beispielhaft und inhaltlich sehr reich ist die WiderstandsBibliographie von Laura Conti, La Resistenza in Italia. 25 luglio 1943 - 25 aprile 1945, Mailand, Feltrinelli, 1961, XV-404 S. 
hundert alle anderen grossen organisierten Fraktionen der politischen Gesellschaft zu bieten haben."1

Zahlreiche Historiker haben diese Sachlage erkannt und selber ihre Arbeitsinstrumente geschaffen, dabei neue Methoden entwickelt und den Rahmen der herkömmlichen Bibliographie erweitert. Prof. Zanna Kormanowa hat uns schon 1935 die „Materialien zur Bibliographie der gedruckten sozialistischen Literatur Polens" gegeben, wobei sie, ohne der Strenge des Historikers etwas zu vergeben, die kleinsten und scheinbar unwichtigsten Details bibliographisch erfasst hat. ${ }^{2}$

Der Commission internationale d'histoire des mouvements sociaux et des structures sociales kommt das grosse Verdienst zu, die Initiative zur Arbeit an dem bekannten Repertorium gedruckter Quellen zur Ersten Internationale genommen zu haben. Damit hat die Kommission nicht nur der Forschung ein Arbeitsinstrument verschafft, sondern auch die Historiker aufmerksam gemacht sowohl auf diese Quellengattung als auf die Wichtigkeit derartiger Bibliographien. Vor allem aber hat der Entschluss der Kommission der Geschichte der Arbeiterbewegung den ihr zukommenden Platz im Studium der modernen Geschichte gesichert und damit erheblich beigetragen zu der seitdem wahrzunehmenden erhöhten Forschungstätigkeit auch in nicht-sozialistischen Ländern. Dies hat sich auch in der zunehmenden Anzahl von Repertorien und Bibliographien ausgedrückt, von denen einige grössere Arbeiten zu Hauptthemen genannt seien: Italienische Arbeiterbewegung (1956-1966), Italienische Arbeiterpresse (1956-1961), KPD (1961), Oesterreichische Arbeiterbewegung (1962), Französische Gewerkschaftsbewegung (1963), ${ }^{3}$ Zweite Internationale (1964).4 Mehrere wichtige Arbeiten sind leider nur polykopiert in geringer Auflage erschienen und zu internem Gebrauch einiger Institute bestimmt, wie die Repertorien der ungarischen Arbeiterpresse, das Marx/EngelsRepertorium der Prager Universität und das Inventar der reichen

1 Siehe S. 7, Anm. 2.

2 Żanna Kormanowa, Materialy do bibliografii druków socjalistycznych na ziemach polskich w latach 1866-1918, Warschau 1935, XVIII-375 S., 2. Aufl. Warschau 1949, XXII-[2]-342-1 S. Von derselben: Materiaty do bibliografii Polskiego ruchu robotniczego (1918-1939), T. I Druki zwarte, Warschau 1960, 580-[1] S.

${ }^{3}$ E.S.M.O.I., Bibliografia del socialismo e del movimento operaio italiano: Periodici [2 Bde] und Libri, opuscoli, articolo, almanacchi, numeri unici [3 Bde], Rom-Turin 1956-1966, 1427 \& 561 \& 673 \& 760 S.; Franco Della Peruta, Bibliografia della stampa periodica operaia e socialista italiana (1860-1926), vgl. S. 10, Anm. 2; Enzo Collotti, Die Kommunistische Partei Deutschlands 1918-1933, Mailand 1961, 217 S.; Herbert Steiner, Bibliographie zur Geschichte der österreichischen Arbeiterbewegung 1867-1918, Wien 1962, 315-[1] S. (ein zweiter Band ist im Druck); Robert Brecy, Le mouvement syndical en France 1871-1921. Essai bibliographique, Paris-Haag, Mouton, 1963, XXXVI-217 S.

1 Georges Haupt, La Deuxieme Internationale (vgl. S. 7, Anm. 2). 
Sammlung Dokumente der $Z$ weiten Internationale, die sich im Warschauer Institut für Parteigeschichte befindet. ${ }^{1}$

Bei den Repertorien sind drei Kategorien zu unterscheiden, die der verschiedenen Natur und Herkunft der repertorierten Dokumente entsprechen: A) Arbeiterpresse; B) Literatur nationaler Organisationen; C) Literatur internationaler Organisationen. Diese grossen Kategorien können unterteilt werden nach den verschiedenen Gebieten, auf denen die Arbeiterbewegung tätig ist (Partei, Gewerkschaft, Genossenschaft usw.). Jedes einzelne Repertorium stellt dem Bibliographen seine besonderen methodischen und technischen Probleme.

A. Bei den Presserepertorien kann die unter der Leitung von Della Peruta begonnene Serie als vorbildlich gelten. ${ }^{2}$ Es ist zu hoffen, dass die aus äusseren Gründen unterbrochene Arbeit wieder aufgenommen wird und vor allem, dass die in ihr angewandten Forschungsmethoden und die Darstellungsart für die Presserepertorien anderer Länder übernommen werden. Auf einige Punkte sei hier besonders hingewiesen: Die bibliographische Beschreibung lässt kein einziges Detail ausser Acht, das Forscher der verschiedensten Spezialgebiete interessieren könnte. Sie vermeldet die Namen der Drucker und der verantwortlichen Herausgeber, der Redakteure (mit genauen Angaben über die Periode ihrer Mitarbeit), ständiger und gelegentlicher Mitarbeiter, die Aenderungen der Periodizität, des Formats, der Seitenzahl und der Auflage. Beschlagnahmte sowohl als Sondernummern sind angegeben. Della Peruta und seine Mitarbeiter haben sich nicht mit dieser ins Einzelne gehenden äusseren Beschreibung begnügt, sondern jedes Organ eingehend analysiert und einzelne Artikel in ihren historischen Zusammenhang gestellt, sowohl hinsichtlich darin zum Ausdruck kommender ideologischer Einflüsse als auch in Hinsicht auf unmittelbar oder später nachweisbaren Einfluss der Artikel.

1 A magyar munkásmozgalmi sajtó bibliográfiája 1848-1948, Budapest 1951, 3 Bde, 473 S. ; idem [Nachtrag zu 1-3], Budapest 1959, [1]-169 S.; idem [Fortsetzung 1949-1956], Budapest 1963, [1]-356 S.; Miroslav Havranek \& Kamil Groh, Soupis spisu a článku Karla Marxe e Bedricha Engelse $z$ fondu Universitni knihovny a Slovanské knihovny v Praze, Prag 1954, VII-[3]-428 S.; Opis materiatow $i$ dokumentow II Międzynarodówki 1889-1914 (w Zakladzie Historii Partii przy $K C P Z P R$ ), Warschau 1962, $90 \mathrm{~S}$.

2 Vgl. S. 9,Anm. 3 für den Titel der Serie. Der Arbeitsplan sah 24 Bände vor, die jeweils die Presse einer Region behandeln sollten. Erschienen sind nur die Teile III, Band 1 \& 2, und XIII, Band 1: I periodici di Milano. Bibliografia e storia. T. 7 (1860-1904) \& T. 2 (1905-1926), Mailand, Feltrinelli, 1956 \& 1961, VIII-363 \& VII-[1]-406-[1] S.; I periodici di Messina. Bibliografia e storia, Mailand, Feltrinelli, 1961, IX-[1]-233 S. 
B. Das Repertorium der aus den verschiedenen politischen und gewerkschaftlichen Organisationen eines Landes hervorgegangenen Dokumente muss dem Forscher erlauben, die wichtigsten Quellen aufzufinden, die unentbehrliche Grundlage jeglicher geschichtlichen Untersuchung der Arbeiterbewegung und des Sozialismus. Bis auf wenige Ausnahmen sind gerade diese gedruckten Quellen bis jetzt noch nicht entsprechend zusammengefasst worden. Eine Analyse der wenigen bereits veröffentlichen Arbeiten dieser $\mathrm{Art}^{1}$ zeigt, mit welchen Problemen sich die Bearbeiter künftiger National-Bibliographien auf unserem Arbeitsgebiet auseinanderzusetzen haben werden und welche Lösungen möglich sind.

Zuallererst stellt sich die Frage: Muss allumfassende Vollständigkeit angestrebt werden oder darf man sich mit einer Auswahl begnügen? Die Gegner der ersten Lösung begründen ihren Standpunkt mit der bequemeren Hantierbarkeit einer Bibliographie, die alle Nachweise und Hinweise auslässt, welche der Verfasser für zweitwichtig oder unwichtig hält oder die er als nicht der nationalen Bewegung zugehörig betrachtet, wie z.B. Uebersetzungen. Diese Beschränkung scheint uns weder die Komplexität der Geschichte mit allen ihren Hintergründen genügend $\mathrm{zu}$ berücksichtigen noch die berechtigten Ansprüche der spezialisierten Forscher, die sie zu ergründen suchen. Ausserdem kann kein Historiker oder Bibliograph voraussehen, was später für Kollegen und Benutzer wichtig oder unwichtig sein wird. Wir können heute nur davon ausgehen, dass einzig die Kenntnis der Gesamtheit aller Veröffentlichungen ein Urteil darüber erlaubt, welche Gedanken in welchem Ausmasse verbreitet wurden, welchen Einflüssen eine Bewegung unterlag und wie sie handelte. Solchen Benutzern, die nur ein Teilgebiet der Gesamtliteratur interessiert, können entsprechend ausführliche Spezialindexe den direkten Weg zu ihren Quellen weisen.

Wenn Vollständigkeit angestrebt werden und keine Auswahl stattfinden soll, so bleibt doch die Frage nach der Begrenzung der aufzunehmenden Schriften. Sie wird für die verschiedenen Länder und Zeitabschnitte unterschiedlich beantwortet werden müssen, aber es empfiehlt sich doch, nach einigen allgemeinen Regeln zu suchen. Selbst-

1 Ausser den bereits erwähnten Arbeiten (S. 1, Anm. 1; S. 4, Anm. 1-4; S. 9, Anm. 2-3) wären noch zu nennen Sergije Dimitrijević, Bibliografija socijalistickog $i$ radnickog pokveta u Srbiji ( $Z$ a osvrtom na ostale krajeve naše zemle), Belgrad, Rad, 1953, 215 S.; vom selben Verfasser Srpska socijalisticka prevodna literatura, Belgrad, Rad, 1958, 144 S.; Radoslav Perovič, Prilog bibliografiji srpskog radničkog pokreta (do 1919), Belgrad, Rad, 1957, 222-[2] S.; Jože Munda, „Bibliografija delavskega socialističnega tiska na Slovenskem od 1868 do 11. aprila 1920", in: Prispevki za Zgodovino Delavkega Gibanja 1962, Nr. 1, S. 1-44; Bibliografie $k$ dejinam ¿eskeho a slovenskeho delničkeho hnuti. 1844-1917, Prag 1965, $383 \mathrm{~S}$. (polykopiert, vom Institut für Parteigeschichte zusammengestellt). 
verständlich müssen alle Schriften erfasst werden, die von Arbeiterorganisationen aller Art ausgehen, von Unterstützungskassen bis zur politischen Partei, und weiterhin die theoretischen und politischen Schriften aktiver Teilnehmer an der Arbeiter- und sozialistischen Bewegung. Dabei ist diese letztere in ihrem weitesten Umfang aufzufassen, von atheistischen Anarchisten bis $\mathrm{zu}$ Christen-Sozialisten. Weiterhin muss überlegt werden, ob oder in welchem Masse Dokumente aufgenommen werden sollen, die von Bewegungen und Strömungen ausgehen, welche den uns hier interessierenden verwandt sind oder doch Berührungspunkte mit ihnen haben, wie z.B. die Volkstümler (Populisten), die Freidenker, gewisse Comtisten und Positivisten (in Lateinamerika, England und Belgien), Kathedersozialisten, Fabier, Tolstoyaner, Bellamyaner, ${ }^{1}$ Esperantisten usw. Schliesslich gibt es

a) Autoren, die sich von der Bewegung zurückziehen oder ausgeschlossen worden sind, aber weiterhin in ihrem Sinne schreiben, wie z.B. Korsch oder Thalheimer;

b) Autoren, welche das Lager gewechselt haben (wie MacDonald und Briand) und eventuell bis zur äussersten Rechten gegangen (wie Alexandre Cohen) oder sogar deren Leiter geworden sind (wie Mussolini und Doriot);

c) Autoren, welche keiner dieser beiden Kategorien zuzurechnen sind, aber eine ideengeschichtlich wichtige Gruppe bilden, wobei wir an Sorel und De Man oder auch an Parvus denken.

Nur die erste Gruppe erlaubt es, eine allgemeine Regel aufzustellen: nicht das Parteibuch des Verfassers, sondern der Zweck und Inhalt einer Schrift entscheiden. In den beiden anderen Fällen muss der Bibliograph, ebenfalls nach einer Prüfung des Inhaltes seiner Schriften, bestimmen, von welchem Augenblick an ein Verfasser nicht mehr der Definition "sozialistischer Schriftsteller" entspricht - auch wenn er sich noch weiterhin auf den Sozialismus beruft, wie z.B. De Man.

Wir haben hier einige Fragen betrachtet, welche sich aus dem inneren Leben der Bewegung ergeben. Andere Fragen entstehen im Zusammenhang mit der historischen Entwicklung:

1) Die allgemeine Emigration aus wirtschaftlichen Gründen, eine Erscheinung sowohl des 19. als des 20. Jahrhunderts, hat die Bildung von Arbeiter- und sozialistischen Organisationen verschiedener Art in jenen Ländern mit sich gebracht, wo die Emigranten sich vorüber-

Sylvia Bowman, Edward Bellamy Abroad. An American Prophet's Influence, New York 1962, 543 S., unterrichtet u.a. über die Beziehungen zwischen der Arbeiterbewegung und den Bellamy-Vereinigungen (besonders S. 208-218, über die Niederlande, wo man heute die grösste Anzahl dieser Vereinigungen antrifft). Eine Bellamy-Bibliographie hat dieselbe Verfasserin veröffentlicht in The Year 2000: A Critical Biography of Edward Bellamy, New York 1958, S. 346-393. 
gehend oder endgültig niederliessen. Die Literatur dieser Organisationen benutzt vor allem die Muttersprache, aber auch die des neuen Vaterlandes, und sie ist immer für die Emigranten selbst bestimmt. Die verwickelten Beziehungen dieser Organisationen sowohl mit der Bewegung des Heimatlandes als mit der des Emigrationslandes schaffen für den Bibliographen ein Dilemma: gehört diese Literatur in das Repertorium des einen oder des anderen Landes, entscheidet die Sprache oder der Druckort? Oder ist für diese Art Literatur ein besonderes Repertorium zu schaffen? Es gibt übrigens bereits eine sehr ausführliche Bibliographie der deutsch-amerikanischen Presse, die sowohl die englisch- als die deutschsprachige und u.a. die sehr reiche sozialistische Presse berücksichtigt. ${ }^{1}$

2) Die Literatur der politischen Emigration wirft keine prinzipiellen Probleme auf. Alle Verfasser nehmen sie in die nationalen Repertorien der Heimatländer auf. Hier entsteht dagegen eine praktische Schwierigkeit, denn die meisten dieser Veröffentlichungen kommen in den klassischen Quellen, wie z.B. in den Nationalbibliographien der Editionsländer, nicht vor. Insbesondere gilt dies für diejenigen Schriften, die zur illegalen Verbreitung im Heimatlande bestimmt waren.

3) Es ist auch vorgekommen, dass eine ganze Literatur ausserhalb des Landes gedruckt worden ist, für das sie bestimmt war, ohne dass hierzu politische Verfolgungen, Polizeimassnahmen oder Verbote gezwungen hätten. So haben z.B. die Serbische sozialistische Partei und die Sozialdemokratische Partei Kroatiens von 1901 bis 1905 eine ganze Anzahl Broschüren in Budapest herausgegeben.

4) Nach Oktober 1917 findet man eine grosse Zahl fremdsprachiger Veröffentlichungen, die in Russland erschienen. Auf derartige Veröffentlichungen internationaler Organisationen wie der Komintern, Profintern oder der Internationalen Roten Hilfe, kommen wir weiterhin zurück. Hier interessiert zuallererst ein Sonderfall, die Veröffentlichungen einzelner Kriegsgefangenen-Gruppen, die sich der Revolution angeschlossen hatten, und die also weder der politischen noch der wirtschaftlichen Emigration zuzuzählen sind. Die Veröffentlichungen können auch nicht bei den internationalen Organisationen untergebracht werden, denn sie erschienen schon vor der Gründung der Komintern. Sie waren ebensowenig für das Heimatland der Kriegsgefangenen bestimmt - wenn sie dort auch einen gewissen Einfluss indirekter Art nach der Rückkehr der Kriegsgefangenen ausüben konnten. Welche Bibliographie muss diese Kategorie erfassen?

5) Dieselbe Frage erhebt sich angesichts der zahllosen Titel in sehr

1 Karl J. R. Arndt \& May E. Olson, German-American Newspapers and Periodicals 1732-1955. History and bibliography, Heidelberg, Quelle \& Meyer, 1961, $794 \mathrm{~S}$. 
vielen Sprachen, welche seit etwa 40 Jahren ein Moskauer Verlag veröffentlicht, der nach verschiedenen Umtaufen neuerdings „ProgressVerlag" heisst. Nur die für kommunistische Parteien im Exil veranstalteten Ausgaben dieses Verlages können der Emigrationsliteratur (2) zugerechnet werden. Nach dem Moskauer Vorbild organisiert, verbreitet seit einigen Jahren auch der Pekinger „Verlag für fremdsprachige Literatur" Ausgaben in mehreren Sprachen.

6) Ein anderes Problem verdient erwähnt und sine ira et studio erwogen zu werden: wohin gehören die Dokumente, welche infolge geschichtlicher Umwälzungen, z.B. Gebietsabtretungen und Grenzverrückungen, das „Vaterland” geändert haben? Muss für den Bibliographen die historische Gegebenheit der Vergangenheit gelten oder die heutige Wirklichkeit? Was wiegt schwerer, die Sprache und die ethnische Zugehörigkeit - oder der politische Begriff des Staates? Verschiedene Autoren haben die Frage verschieden beantwortet.

Schliesslich ist ein Problem von sehr weittragender Bedeutung zu erwähnen, dem eine Spezialuntersuchung zu widmen wäre. Es ist dies das Problem der nichtsozialistischen revolutionären Bewegungen, die mit der Arbeiterbewegung zusammengehen, vom Sozialismus beeinflusst werden oder sich sogar direkt auf ihn berufen. Bereits die europäische Geschichte des 19. Jahrhunderts ist reich an Beispielen, wie die Anhänger Mazzinis und Garibaldis, sowie bestimmte Gruppen der polnischen Emigration, die alle zeitweise enge Berührung mit der Ersten Internationale hatten. Die Rolle der Anhänger Mazzinis in der Gründungsperiode der Internationale ist ebenso bekannt wie die Verbrüderung von Garibaldianern und Internationalisten in Italien, nach dem Falle der Kommune, oder das Eintreten des Generalsrats für die Fenier, deren Nachfolger im 20. Jahrhundert (James Connolly), die Katholiken, die irischen Nationalisten und die Sozialisten für sich beanspruchen.

Die Zweite Internationale musste sich mit den nationalistischen georgischen Sozialisten, der armenischen Daschnak Partei und schliesslich mit den zionistischen Sozialisten auseinandersetzen. Mit dem Anfang des 20. Jahrhunderts nimmt das Problem Weltausmasse an: zuerst mit dem Erwachen Asiens, dann mit der Erweiterung des Befreiungskampfes der Kolonialvölker, bis endlich heute der Begriff "Sozialismus" bei einer ganzen Reihe neuerdings in die Geschichte eingetretener Völker Aufgeld tut, ohne sich immer mit dem überkommenen Inhalt zu decken.

Der Forscher-Bibliograph der Arbeiterbewegung und des Sozialismus steht vor der Frage, ob er die Veröffentlichungen solcher Organisationen berücksichtigen soll, wie die des während der Revolution von 1908 bis 1911 in Iran gegründeten „Mudjahid”, oder der „Persischen Sozial- 
demokraten, Verteidiger des Islams", oder die Schriften mehrerer muselmanischer Reformatoren, die sich mit dem Sozialismus beschäftigen. Der Gründer der ersten türkischen sozialistischen Organisationen, Hussein Hilmi, war ein ehemaliger Polizeioffizier. Er gab die ersten sozialistischen Zeitschriften in türkischer Sprache heraus, die neben völlig wirren Artikeln auch authentisch sozialistische Texte enthalten - obwohl Hilmis Biograph ihm bescheinigt: „Er kannte vom Sozialismus nicht einmal das S." Gehören Hilmis Zeitschriften in eine Sozialismus-Bibliographie? Was machen wir mit Sun Yat Sen, der sich für den Sozialismus erklärt hat, oder mit Tilak, der in seinen Schriften eine radikale, den sozialistischen Gedanken sehr nahestehende Theorie entwickelt aber sich praktisch-politisch im Rahmen der indischen Kongress-Partei betätigt? Wohin gehören die Schriften Franz Fanons, des Theoretikers der Revolution der Kolonialvölker?

\section{Von den Repertorien gedruckter Dokumente internationaler Organi-} sationen kennen wir nur die der Ersten und Zweiten Internationale sowie zwei vorbereitende Arbeiten zu dem der Dritten Internationale. Die mit ihnen gemachten Erfahrungen geben Anlass zu einigen Bemerkungen über die angewandten Methoden und Kriterien sowie über die Struktur, welche dieser Repertorien-Kategorie am besten entsprechen. Ganz allgemein gilt, dass das Zusammenstellen von Repertorien dieser Art eine intime Kenntnis des Aufbaus und des inneren Räderwerks, der institutionellen Verflechtung der betreffenden Organisation erfordert. Sie ermöglicht festzustellen, welche Dokumente als offiziell zu gelten haben und wie sie entsprechend dem Organisationsaufbau einzuordnen sind. Um dem Forscher sowohl ein handhabliches Arbeitsinstrument als grösstmögliche Information zu bieten, sollten die Dokumente systematisch gruppiert und jeweils durch einleitende $Z$ wischentexte, textkritische und ergänzende Anmerkungen erläutert werden. Die Zusammensteller der vorliegenden Repertorien haben diese allgemeinen Regeln unterschiedlich angewandt.

Das dreibändige Repertorium der Ersten Internationale ${ }^{1}$ ist schon

${ }^{1}$ Repertoire international des sources pour l'etude des mouvements sociaux aux XIXe et XXe siècles. La Première Internationale, Bd. I: Périodiques 1864-1877; $\mathrm{Bd}$. II: Imprimés 1864-1876. Actes officiels du Conseil Général et des Conférences de l'Association Internationale des Travailleurs; Bd. III: Imprimés 1864-1876. Actes officiels des Fédérations et Sections Nationales de l'Association Internationale des Travailleurs, Paris, Colin, 1958-1963, 3 Bde, XX-81-[2] \& 86-[1] \& XIX-[1]223-[1] S. Die neuere Literatur über die Internationale ist in Titellisten zusammengestellt worden von Maximilien Rubel, (Etudes de Marxologie 1964 \& 1965 , Nr. 8, S. 251-275 und Nr. 9, S. 255-260) und Jacques Rougerie (Mouvement Social 1965, Nr. 51, S. 127-138); sie ist in zwei ausgezeichneten Literatur-Ubersichten kritisch behandelt worden von Gian Mario Bravo (Rivista Storica del Socialismo, Florenz, 1965, Nr. 24, S. 3-51) und von F. Svátek (Přispěvky k Dějinám KSĆ, Prag 1965, Nr. 5, S. 793-827). 
vor einigen Jahren abgeschlossen worden, gerade rechtzeitig zu ihrer Hundertjahrfeier, aus deren Anlass man sich naturgemäss häufiger mit dem Repertorium beschäftigt hat, als sonst vielleicht der Fall gewesen wäre. Zahlreiche Besprechungen haben seine unbezweifelbaren Verdienste hervorgehoben, auf die wir deshalb hier nicht näher einzugehen brauchen. Die Gliederung der Dokumente in drei Bände (Presse, Generalrats- und Kongressdokumente, Föderations- und Sektionsdokumente) entspricht der Struktur der Internationale, im Inneren der Bände selbst jedoch ist dieses Prinzip nicht konsequent durchgeführt worden. Die Klassifikation der erfassten Dokumente nach ideologischen Gesichtspunkten ist von vielen Kritikern abgelehnt worden. Anstatt sich auf den Boden der historischen Wirklichkeit zu stellen, in der bis 1872 eine einzige Organisation Vertreter verschiedener Tendenzen umfasste und die seit 1873 in zwei getrennten Organisationen gleichen Typus weiterbestand, übernimmt die Redaktion ein Nachwelturteil, das die Vertreter zweier Tendenzen einander gegenüberstellt und sich dabei für die eine und gegen die andere ausspricht. Die Anwendung dieses ideologischen Kriteriums hat in dem der Presse gewidmeten ersten Band zwei Chronologien zur Folge, und sie führt im zweiten Band zum Ausschluss der Kongressdokumente jener föderalistischen Organisation, die seit 1873 selbständig neben der ursprünglichen Internationale bestand, deren Namen sie ebenfalls führte. Die Redaktion hat einige dieser Dokumente im Anhang zum dritten Bande untergebracht, der Föderations- und Sektions-Dokumente enthält. Dieser Band enthält übrigens eine ganze Anzahl Titel, deren Aufnahme ungerechtfertigt erscheint, da die herausgebenden Arbeitervereine niemals der Internationale angehört haben.

Der zweite Band desselben Repertoriums gibt Anlass zu einer anderen Frage prinzipieller Art: soll der Bibliograph beim Erfassen offizieller gedruckter Dokumente einer Organisation im engen Rahmen der Bibliothekspraxis bleiben oder muss er die Kriterien des Historikers anwenden? Mit anderen Worten, darf er nur solche Dokumente aufnehmen, die als selbständige Druckschrift (Buch, Broschüre oder Flugblatt) erschienen sind oder muss er auch die ihrer Natur und ihrem Ursprung nach gleichartigen Dokumente (Adressen, Zirkulare, Erklärungen) verzeichnen, die nur in der Arbeiterpresse erschienen sind? Die Redakteure des Repertoriums der Ersten Internationale haben sich für die erstgenannte Auffassung entschieden und nur selbständige Druckschriften aufgenommen. In der Einleitung erklären sie: „Die in diesem Bande zusammengestellten Dokumente machen es möglich, die Tätigkeit der leitenden Organe [der Internationale] zu verfolgen." Der Band II beweist, dass dieser Zweck mit der hier angewandten Methode nicht erreicht werden kann. Das Repertorium führt insgesamt 
ungefähr 50 offizielle Dokumente des Generalrats von 1864 bis 1875 an, die als selbständige Druckeinheit erschienen. Damit sind mindestens ebenso viele Dokumente gleicher Natur und desselben Ursprungs ungeachtet ihrer Wichtigkeit ausgeschlossen worden, mit der einzigen „Begründung”, dass sie nur in Zeitschriften veröffentlicht worden sind. Von diesen fehlenden Tätigkeitszeugnissen des Generalrats nennen wir nur: die Adresse an Lincoln (vom Dezember 1864), die Erklärung zugunsten der Unabhängigkeit und Integrität Polens (vom März 1865), die Adresse an Johnson (vom Mai 1865, anlässlich Lincoln's Ermordung) und die an das amerikanische Volk (vom September 1865), sowie die an den englischen Innenminister gerichtete Denkschrift zugunsten von vier zum Tode verurteilten Feniern (vom September 1867). Von den zahlreichen, die Kommune betreffenden Dokumenten sind nur die Adresse über den Bürgerkrieg in Frankreich und der Protest gegen den Botschafter Washburne aufgenommen worden. Ein derartiges Editionsprinzip muss umso befremdlicher erscheinen, als die Presseveröffentlichungen des Generalrats im allgemeinen ein viel grösseres internationales Publikum hatten, als Flugblätter und Broschüren. Uebrigens ist sich die Redaktion dieser Tatsache auch bewusst gewesen, wenn sie für einzelne der aufgenommenen Dokumente in einer Anmerkung gleichzeitige Abdrucke in der Presse nachweist.

Das Repertorium der Dokumente der Zweiten Internationale ${ }^{1}$ beschäftigt sich ausschliesslich mit solchen Dokumenten, welche von den Kongressen und Konferenzen ausgingen oder an sie gerichtet wurden, und mit den Dokumenten des Büros der Sozialistischen Internationale. Mehrere ausführliche Einleitungen unterrichten über die Quellenlage, die Bedeutung, welche den verschiedenen DokumentenGruppen zukommt, und über die vom Verfasser angewandte Methode, wobei auch Fragen der bibliographischen Methode im allgemeinen berührt werden. Die Arbeit wurde 1964 abgeschlossen, und der Verfasser hat bereits die kritischen Bemerkungen berücksichtigen können, welche das Repertorium der Ersten Internationale hervorgerufen hat. So sind die, allerdings verhältnismässig wenigen, nur in der Presse veröffentlichten Dokumente miterfasst worden, und ausserdem hat der Verfasser in Einzelfällen, wenn kein Exemplar der selbständigen Veröffentlichung eines Dokumentes gefunden werden konnte, seine Veröffentlichung in der Presse nachgewiesen. Derart ist die annähernde Vollständigkeit des Repertoriums erreicht worden, welche dem ersten Repertorium fehlt. Wie im ersten Repertorium, sind auch im zweiten die Standorte der Dokumente nachgewiesen, jedoch im Gegensatz zum

1 Von Georges Haupt, zitiert S. 7, Anm. 2. Die Dokumente der internationalen Kongresse von 1877 bis 1888 sind repertoriert von Georges Haupt und Jeannine Verdès in Mouvement Social 1965, Nr. 51, S. 113-126. 
ersten gibt das zweite Repertorium nur jeweils einen Standort an, die Bibliothek oder Sammlung, wo der Verfasser das Dokument benutzt hat. Damit wird dem Forscher die Arbeit erschwert, und das gleiche gilt für die hier fehlenden Nachweise von Nachdrucken in der zeitgenössischen Presse. Ausserdem sind alle Dokumente nur in den drei offiziellen Verhandlungssprachen der Zweiten Internationale nachgewiesen, Deutsch, Englisch und Französisch, während die meisten auch auf italienisch, spanisch, dänisch usw. erschienen.

Die grossen internationalen politischen und gewerkschaftlichen Organisationen der Zwischenkriegszeit haben ihre Repertorien noch nicht erhalten. Für die Dritte Internationale allerdings stehen bereits zwei bedeutende Vorarbeiten zur Verfügung, nämlich die Auswahlbibliographie von G. Proccaci ${ }^{1}$ und ein sehr reichhaltiges Inventar, das W. Sworakowski veröffentlicht hat. ${ }^{2}$ Die künftigen Bibliographen und Forscher werden die Erfahrungen ihrer Vorgänger nützlich verwerten können, aber sie werden sich auch vor heikle Probleme gestellt finden, wie im Falle der Dritten Internationale, deren Archive bis jetzt völlig unzugänglich sind. Wo anders aber soll der Forscher feststellen, in welchem Masse anderwärtig zugängliche Sammlungen von KominternDokumenten vollständig sind? Weiterhin, sollen nur die offiziellen Dokumente der Kongresse und des Exekutiv-Komités aufgenommen werden oder auch die wichtigsten Dokumente der einzelnen Sektionen? Und wie deren Wichtigkeit ermessen? Sollen die Massenorganisationen miteinbezogen werden und in welchem Masse? Einige waren sehr eng mit der Komintern verbunden, wie z.B. die Kommunistische Jugendinternationale, andere wurden von ihr nur in verschiedenem Masse beeinflusst, z.B. die International of Seamen and Harbourworkers.

4. Die "spezialisierten Bibliographien" bezeichnen wir so im Gegensatz zu den allgemeinen Bibliographien und Repertorien, von denen bisher die Rede gewesen ist. Ihre jeweilige Besonderheit bringt für die Bibliographien dieser Kategorie eine Vielzahl ihnen ganz eigener Probleme mit sich, während sie nur wenige gemein haben. Die gleichen Probleme nehmen in jedem Einzelfall eine andere Form an. Die jeweilige Besonderheit ihres Gegenstandes bestimmt meistens schon die Antwort auf die erste Frage, welche bei der Arbeit an seiner Biblio-

1 Giuliano Procacci, „L'Internazionale Comunista dal I al VII Congresso, 19191935", in: Annali, Jg. I, S. 283-350.

2 Witold S. Sworakowski, The Communist International and its Front Organisations. A research guide and checklist of holdings in American and European Libraries, Stanford, Hoover, 1965, $493 \mathrm{~S}$. Die Komintern ist auch der Gegenstand einer Bibliographie von Vlasta Krupickova, Soupis literatury k déjinám Komunistické Internacionaly, Prag 1964, $120 \mathrm{~S}$. 
graphie auftaucht: soll sie ein einfaches Inventar sein oder eine repräsentative oder eine Auswahlbibliographie, oder soll sie vollständig sein? Aber welche Kriterien bestimmen den Repräsentativ-Charakter eines bibliographischen Elementes, und nach welchen Gesichtspunkten auswählen? Wo fängt die Vollständigkeit an? Denn sie hat mehrere Dimensionen, geht von der einfachen Gesamtliste der Erstdrucke aller Dokumente (der wichtigsten und der unbedeutendsten) bis zur teilweisen (für eine bestimmte Periode oder für bestimmte Sprachen) oder vollständigen Aufnahme aller Nachdrucke und Uebersetzungen, oder sogar der Berücksichtigung von Zitaten. Es kommt sogar vor, dass Manuskripte mitaufgenommen werden müssen, wie wir weiterhin zeigen werden. Die jeweilige Besonderheit des Gegenstandes und der besondere Zweck jeder einzelnen Bibliographie werden auch zu entscheiden haben über ihre Gliederung und über den Umfang und den Charakter von erläuternden Anmerkungen usw.

Wir unterscheiden drei Arten spezialisierter Bibliographien, nach ihrem allgemeinen Gegenstande: A) Das Gesamtwerk eines einzigen Autors (z.B. Marx); B) ein Einzelwerk (z.B. „Das Kapital”); C) ein in der Zeit oder vom Gegenstand her begrenztes Thema (z.B. Veröffentlichungen der SPD während des Ersten Weltkrieges oder Marx über Kolonialismus).

A. Das Gesamtwerk einzelner sozialistischer Autoren ist schon seit längerer Zeit Gegenstand selbständiger bibliographischer Arbeiten unterschiedlichen Wertes und verschiedener Konzeptionen. Uebrigens gibt es keinen Prototyp. Die Gliederung der Bibliographie, die aufzunehmenden Elemente, Umfang und Einzelheiten der Darstellung hängen nicht nur von der objektiven Wichtigkeit des Werkes und des Autors ab, die Gegenstand der Bibliographie sind, sondern auch von dem $Z$ weck, den sie verfolgt, und den herangezogenen Quellen. Die einer Bibliographie in optima forma für würdig gehaltenen Autoren sind selbstverständlich die überragenden grossen Persönlichkeiten, wie Marx und Engels, Proudhon und Lenin und andere, deren Ideen und Handeln eine Epoche beeinflusst und geprägt haben, oder auch solche, die als anerkannte Repräsentanten dieser Ideen sie interpretiert und verbreitet haben, wie z.B. Kautsky.

Hier berühren wir übrigens den Hauptgrund, der nach unserer Auffassung den Bibliographen der Arbeiterbewegung und des Sozialismus dazu berechtigt, völlig mit den Regeln der klassischen Bibliographie zu brechen. Die grosse Mehrheit der Persönlichkeiten, deren Werke ihn beschäftigen, und praktisch alle Organisationen, haben nicht nur Ideen formuliert und veröffentlicht, sondern vor allem praktisch gehandelt. Idee und Aktion bilden bei ihnen eine Einheit. So sind im 19. Jahr- 
hundert beispielsweise der Erstdruck und jede Uebersetzung oder Verbreitung des Kommunistischen Manifestes nicht nur in bibliographischen Daten erfassbare Drucksachen, sondern politische Handlungen, die nachweisbare politisch-organisatorische Gründe, Begleitumstände, Zwecke und Folgen haben. ${ }^{1}$

Man könnte die oben angeführten Namen mit einer langen Liste von mehr oder weniger berühmten Persönlichkeiten fortsetzen, die auf der internationalen oder auf nationaler Ebene eine mehr oder weniger bedeutende Rolle gespielt haben. ${ }^{2}$ Wir wählen aus den ihren Werken gewidmeten Bibliographien einige aus, die hier durch ihre Konzeption oder die angewandten Methoden und Kriterien interessieren können.

Die vollständige Bibliographie der Werke von Marx und Engels lässt immer noch auf sich warten. Wir werden uns hier nicht mit den vielen und grossen Schwierigkeiten beschäftigen, die mit dazu geführt haben, dass wir noch wohl längere Zeit ohne ein derartig wichtiges Arbeitsinstrument auskommen müssen. Als erster Versuch einer systematischen Marxbibliographie darf Huberts „Bibliographische Einführung" gelten, die als Anhang einer Untersuchung von Henri Desroche 1950 erschien. ${ }^{3} \mathrm{Er}$ hat als erster mit den herkömmlichen bibliographischen Auffassungen und Regeln gebrochen, eigene Formeln angewandt und seine Bibliographie bereichert durch zahlreiche Synopsen, Angaben über die Genesis einzelner Schriften, Nachweis von Uebersetzungen usw. Huberts Arbeit ist heute zwar in vielen Punkten

1 Vgl. Bert Andréas, Le Manifeste Communiste de Marx et Engels. Histoire et Bibliographie 1848-1918, Mailand, Feltrinelli, 1963, VIII-429-[5]-23 S.

2 Von Vorläufer-Bibliographien seien genannt: Giuseppe Del Bo, Charles Fourier e la Scuola Societaria (7801-1922). Saggio Bibliografico, Mailand, Feltrinelli, 1957, [4]-111 S. ; ,Bibliographie raisonnée et critique” Babeufs in Maurice Dommanget, Pages choisies de Babeuf usw., Paris, Colin, 1935, S. 5-42 (eine erweiterte und verbesserte Fassung hat der Autor inzwischen abgeschlossen); eine WeitlingBibliographie in Gian Mario Bravo, Wilhelm Weitling e il comunismo tedesco prima del Quarantotto, Turin, Giappichelli, 1963, S. 343-357. Ueber Lassalle: Bert Andréas, „Verlagsgeschichtliches 1865-1878” und „Bibliographie der Schriften von Ferdinand Lassalle und Auswahl aus der Literatur über ihn", in: Archiv für Sozialgeschichte, Hannover 1963, Jg. III, S. 322-423. Eine Luxemburg-Bibliographie wird in Polen vorbereitet, die Erstdrucke sind erfasst in Jadwiga Kaczanowska \& Feliks Tych, „Bibliografia pierwodruków Rozy Luksemburg”, in: Z Pola Walki, Warschau 1962, Nr. 3, S. 161-226.

${ }^{3}$ Charles-Fr. Hubert [Pseudonym von Guillaume Dunstheimer], „Initiation bibliographique à l'œuvre de Marx et d'Engels, et au marxisme", in: $H$. C. Desroche, Signification du marxisme, Paris, Ed. ouvrières, 1950, S. 249-377. Eine Literaturübersicht von mehr als 1000 Titeln wurde zusammengestellt von E. Czóbel und P. Hajdu, „Die Literatur über Marx, Engels und über Marxismus seit Beginn des Weltkrieges (Mit Ausnahme der russischen) (Mit der Beilage: Die Lassalle-Literatur derselben Periode)", in: Marx-Engels Archiv, Frankfurt a.M. [1926], Bd. I, S. 467-549. 
überholt, aber sie bleibt bemerkenswert und ist damals leider so gut wie unbeachtet geblieben, vielleicht, weil das Buch des Katholiken Desroche trotz des ursprünglich erteilten nihil obstat bald durch Zwischenkunft der Kirchenbehörden aus dem Handel gezogen wurde.

Den ersten Versuch, eine Marxbibliographie nach wissenschaftlicher Methode zusammenzustellen und mit Hilfe des ganzen modernen Forschungsapparates, stellt die 1956 erschienene Arbeit Maximilien Rubels dar, der 1960 ein ziemlich unfangreicher Band mit Berichtigungen und Ergänzungen folgte. ${ }^{x}$ Diese beiden Bände bilden die einzige annähernd vollständige Marxbibliographie wissenschaftlichen Charakters, über die wir verfügen, denn das 1957 von Gertrud Hertel veröffentlichte "Inhaltsvergleichsregister" ist ein reines Titel-Inventar. ${ }^{2}$ Die Arbeit Rubels bleibt also vorläufig ein unentbehrliches Arbeitsinstrument, $\mathrm{zu}$ dem hier nur einige allgemeine Bemerkungen gemacht werden sollen. Rubel bekennt sich schon im Titel seiner Arbeit deutlich zu einer Auffassung, die er in der Einleitung ausführlich darlegt. Diese Auffassung unterscheidet zwischen Marx, dem „Schöpfer einer selbständigen Soziologie-Lehre”, und Engels, dem einfachen „Interpreten und Verbreiter der Marxschen Theorien". Diese Auffassung sieht in Engels den ,eigentlichen Urheber des ,Marxismus', mit anderen Worten, der Systematisierung und Schematisierung einer Lehre, die als das gerade Gegenteil jeglicher Ideologie gedacht war". Gegen diese Auffassung sind zwei Einwendungen zu erheben. Die gegenseitige Durchdringung des Marx-Engels'schen Gedankengutes und die weitgehende Identität ihrer Auffassungen nach gemeinsamer fortwährender ,,Selbstverständigung" werden von den meisten Forschern angenommen und seit 1845 datiert, als Marx und Engels zuerst eine Veröffentlichung gemeinsam unterzeichneten. Diese Einheit der Marx-Engels'schen Auffassungen haben bereits manche ihrer Zeitgenossen erkannt und ihre Feststellung tut Karl Marx' unbestreitbarem Genie keinerlei Abbruch. Auch Genies lernen von anderen, und es ist dies vielleicht nicht ihre geringste Qualität. Die von Rubel vertretene Ansicht scheint uns ausserdem Marx und Engels zu sehr auf ihre blosse Bedeutung für die Gesellschaftswissenschaft $z u$ beschränken, denn sie berücksichtigt nicht ihre Tätigkeit seit 1847 innerhalb einer Bewegung, die sie mitbegründet und organisiert haben. Wenn sie mit ihren Schriften der

1 Maximilien Rubel, Bibliographie des auvres de Kavl Marx, avec en appendice un répertoire des auvres de Friedrich Engels und Supplément a la Bibliographie des auvres de Karl Marx, Paris, Rivière, 1956 \& 1960, 272-[1] \& 74-[3] S.

${ }^{2}$ Gertrud Hertel, Inhaltsvergleichsregister der Marx-Engels-Gesamtausgaben, Berlin 1957, XX-295 S. Es ist inzwischen überholt und verbessert durch das Marx/Engels-Verzeichnis. Werke. Schriften. Artikel, Berlin, Dietz, 1966, 27*-[1]$358 \mathrm{~S}$., das besonders nützliche Hilfsregister enthält. 
Arbeiterbewegung eine Theorie gegeben, sie befruchtet und belebt haben, so hat andererseits die Bewegung auch manche dieser Schriften (z.B. programmatische und aufklärende) ausdrücklich von Marx und Engels verlangt und andere (meistens kritische) durch ihre Aktion unmittelbar hervorgerufen. Wir brauchen uns nur der Entstehungsgeschichte von Schriften wie z.B. des Kommunistischen Manifestes, des Anti-Dühring oder der Kritik des Gothaer Programmes zu erinnern. Zur Arbeitsmethode Rubels muss gesagt werden, dass er sich in manchen Fällen zu sehr auf Vorgänger, Bibliographen und vor allem Herausgeber von Marx/Engels Texten verlassen und von ihnen einzelne Irrtümer, z.B. bei Zuschreibungen, übernommen hat. Es sollte aber nicht vergessen werden, dass Rubel Pionierarbeit zu leisten hatte, und dass dabei für ihn vor etwa 15 Jahren die Quellenlage bedeutend ungünstiger war als sie sich heute einem vergleichbaren Unternehmen darstellen würde, dem u.a. die reiche seitdem erschienene Literatur zur Verfügung stehen würde. Zu der Frage irriger Zuschreibungen sei noch an den bekannten Fall jener zahlreichen und wichtigen in der New York Tribune erschienenen Artikel erinnert, als deren Verfasser Marx galt, bis im Jahre 1913 die Veröffentlichung seines Briefwechsels mit Engels den letzteren als den tatsächlichen Alleinverfasser auswies. Bis dahin hatte keiner der berufenen Autoritäten an Marxens Verfasserschaft gezweifelt, und als Kautsky seine eigene Uebersetzung eines Teils der Artikel einleitete, pries er „Marx' historischen Blick”, den diese Artikel bewiesen, und den "die Zeit eines halben Jahrhunderts $[. .$.$] in fast$ allen Punkten bestätigt hat". ${ }^{1}$ Tatsächlich änderte seine erwiesene Nichtverfasserschaft dieser Artikel durchaus nicht das bestehende Marxbild. Von den 10 Manuskripten, welche die Deutsche Ideologie bilden, sind 8 in Engels' Handschrift, nur eines (die Vorrede) in Marxens und eines in Weydemeyers. Der tatsächliche Anteil des einen oder des anderen der beiden Verfasser ist hieraus nicht abzulesen, und Marx spricht 1847 denn auch von der „von Fr. Engels und mir gemeinschaftlich verfassten Schrift". Die hier angeführten Beispiele könnten vermehrt werden. Sie scheinen uns gegen die Rubelsche Auffassung und zugunsten einer Marx/Engels-Bibliographie zu sprechen, die in einer einzigen Chronologie die Schriften beider Autoren erfasst und dabei so genau wie möglich den Nachweis erbringt, welchen Anteil der eine und der andere an der Redaktion der einzelnen Schriften hatte.

Wir haben im vorhergehenden eine wichtige Quelle bibliographischer Forschung erwähnt, Manuskripte und Briefwechsel. Verschiedene Verfasser neuerer Arbeiten haben sie zum Vorteil ihrer Arbeit verwertet, und einer hat sie sogar vollständig inventarisiert und dies auch im ${ }^{1}$ Karl Marx, Revolution und Kontre-Revolution in Deutschland. Ins Deutsche übertragen von K. Kautsky, Stuttgart, Dietz, 1896, S. XX. 
Titel zum Ausdruck gebracht. ${ }^{1}$ Die klar gegliederte und intelligent annotierte Bibliographie gibt die Quellen für die Zuschreibung anonymer Texte, den Standort der Veröffentlichungen und den der nachgewiesenen Hessmanuskripte sowie ihn betreffender Dokumente an. Die sehr zahlreichen Artikel sind jeweils nach den Zeitschriften in denen sie erschienen gruppiert. Da Hess zwischen 1855 und 1860 an sechs verschiedenen Zeitschriften (davon zwei Tageszeitungen) mitgearbeitet hat, ist für die angegebene Periode die Chronologie sechsmal unterbrochen und wiederaufgenommen worden. Vielleicht wäre eine einzige Chronologie vorzuziehen gewesen, die auch die Zusammenhänge zwischen den in den verschiedenen Zeitschriften gleichzeitig behandelten Themen deutlicher gemacht hätte. Die vom Verfasser selber und die von Wolfgang Mönke nachträglich veröffentlichten Ergänzungen tun der bemerkenswerten Vollständigkeit dieser Bibliographie keinen Abbruch. ${ }^{2}$ Dagegen erstaunt das befremdende Fehlen jeglichen Indexes. Die Arbeit interessiert übrigens besonders durch den Platz, welchen sie im Werke des Historikers Silberner einnimmt. Er beschäftigt sich seit etwa 20 Jahren mit Hess, über den er einen ersten bibliographischen Versuch 1951 veröffentlicht hat, dem dann 1958 die hier besprochene vollständige Bibliographie folgte, und 1959 die Ausgabe von Hess' Briefwechsel. Und erst nachdem er sie dieser Art logisch vorbereitet hatte, nahm Silberner die Hess-Biographie in Angriff, die er kürzlich veröffentlicht hat. ${ }^{3}$

Für seine Bibliographie Kautskys ${ }^{4}$ hat Werner Blumenberg ebenfalls dessen reichhaltige Archive benutzt, z.B. um Kautsky als Verfasser zahlreicher anonymer und pseudonymer Schriften zu identifizieren, aber im Gegensatz zu Silberner hat Blumenberg darauf verzichtet, ein Inventar der Archive zu veröffentlichen. ${ }^{5}$ In seiner Einlei-

1 Edmund Silberner, The works of Moses Hess. An inventory of his signed and anonymous publications, manuscripts and correspondence, Leiden, Brill, 1958, XVII-[1]-125-[1] S.

2E. Silberner, „Zur Verfasserschaft der ,Berichte aus Deutschland' in der Genfer Espérance” und „Neues Material zur Geschichte der Genfer Espérance”, in: International Review of Social History, 1962 (Bd VII, S. 441-445) und 1963, (Bd. VIII, S. 447-458). Wolfgang Mönke, „Über die Mitarbeit von Moses Hess an der ,Deutschen Ideologie"', in: Annali, 1963, Jg. VI, S. 438-509, und Mönke, Neue Quellen zur Hess-Forschung, Berlin, Akademie, 1964, 117-[1] S.

${ }^{3}$ Edmund Silberner, Moses Hess. Geschichte seines Lebens, Leiden, Brill, 1966, XVIII-691-[1] S.

- Werner Blumenberg, Karl Kautskys literarisches Werk. Eine bibliographische Ubersicht, Haag, Mouton, 1960, $158 \mathrm{~S}$. Vgl. auch die Besprechung von Bert Andréas in Annali, 1961, Jg. IV, S. 689-702.

5 Ein anderes Beispiel von Archivbenutzung für eine Bibliographie bietet die Kollektivarbeit ChronologiCeskij ukazatel' proizvedenij V. I. Lenina. Knigi, stat'i, vystuplenija, pis'ma i drugie dokumenty, Moskau, IML, 1959-1962, 2 Bde, X-702 
tung gibt er genau an, in welchem Masse er die Archive benutzt hat und erklärt, ihr Inventar würde ebensoviel Platz einnehmen wie die eigentliche Bibliographie. Er erklärt weiterhin, auf die Aufnahme einer Liste dieser Manuskripte und Briefschaften, sowie von Anmerkungen zu den inventarisierten Schriften auf Grund der Archive, verzichtet zu haben, um den Rahmen der Bibliographie nicht zu sprengen. Angesichts der Bedeutung, die Kautskys Schriften und seinem Handeln für die europäische sozialistische Bewegung über die mehr als 60 Jahre seines politischen Lebens hinaus zukommt, ist es zu bedauern, dass Blumenberg gemeint hat, sich zu dieser Beschränkung entschliessen zu müssen. Dies umso mehr, als er durch seine Kenntnisse und den Platz, den er einnahm, als der einzig berufene Verfasser einer über den klassischen Rahmen hinausgehenden vollständigen Bibliographie des Sozialisten Kautsky zu gelten hatte. Es ist übrigens sicher, dass Blumenberg nicht allein verantwortlich für seinen Entschluss war. Er begann die Arbeit an seiner Bibliographie zu einem Zeitpunkt, als das Institut welches Kautskys Archive beherbergt, mehr noch als heute einer Politik vorsichtiger Bescheidenheit huldigte, die es ihm nicht gestattete, seine Schätze öffentlich bekanntzumachen und damit das Interesse der Forscher und ihren befürchteten Sturm auf seine Archive hervorzurufen. Dieselbe Vorsicht hat es Blumenberg ebenfalls untersagt, eine ursprünglich vorgesehene Liste von Kautskys Korrespondenten im Anhang aufzunehmen. Jedoch durch die (in der Einleitung erläuterte) Wahl der Arbeitsinstrumente, durch die klare Gliederung des Stoffes, die weitgehende Berücksichtigung von Uebersetzungen und durch die nach Namen, Themen und Sprachen unterteilten Indexe hat Blumenbergs Bibliographie schon jetzt beispielgebend für mehrere neuere Arbeiten gewirkt.

Dies gilt z.B. für die Bebel-Bibliographie von Ernst Schraepler, ${ }^{1}$ die ausserdem noch eine kurze Chronologie mit den wichtigsten Lebensdaten Bebels enthält, sowie zwei ebenfalls sehr nützliche weitere Indexe der erwähnten Zeitschriften und der Verfasser von Schriften über Bebel. Der Namen-Index gibt ausserdem einige biographische Hinweise.

Die Verfasser der betrachteten Arbeiten haben die anfangs ange-

S. \& VIII-696. Die einzelnen Schriften sind chronologisch nach ihrem Entstehungsdatum (das angegeben wird) geordnet, ihr Erstdruck und die Abdrucke in den ersten vier Ausgaben der Werke Lenins wird nachgewiesen (die fünfte Ausgabe war beim Erscheinen noch nicht abgeschlossen). Ausserdem wird mitgeteilt, ob und wo ein Manuskript, eine Kopie o.ä. vorhanden ist. 1963 erschien ein Band mit zusätzlichen „Hilfsregistern": Vspomogatel'nye Ukazateli k chronologiðeskomu ukazatelju proizvedenij V. I. Lenina, Moskau, IML, 1963, X-490 S.

1 Ernst Schraepler, August Bebel Bibliographie, Düsseldorf, Droste, 1962, $169 \mathrm{~S}$. 
führten Probleme, und noch einige andere, auf verschiedene Arten gelöst. Fast alle haben mehr oder weniger die gleichen allgemeinen Quellen benutzt, aber nur einer hat sich auch den öffentlichen Archiven zugewandt. Die Historiker des Sozialismus und der Arbeiterbewegung benutzen seit 50 Jahren in immer zunehmendem Masse die Staats- und Polizei-Archive, und die Bibliographen desselben Gebietes täten gut daran, sich ebenfalls dieser reichhaltigen und interessanten Quellen zu erinnern. Das Interesse der unterschiedlichsten Bibliographie-Benutzer wird in dem Masse zunehmen, wie die Bibliographen es ansprechen und anregen, indem sie z.B. in den spezialisierten Bibliographien ihre Arbeiten durch Elemente und historische Fakten aus allen zugänglichen Quellen bereichern.

Wenn auch die Bibliographien der Werke sozialistischer Verfasser zahlreich sind, so bleibt doch noch viel zu tun, und selbst manche wichtigen Lücken sind noch zu füllen. Wir haben bereits den Fall Marx/Engels erwähnt. So weit wir wissen, fehlt auch eine grössere Bibliographie z.B. Proudhons, die es erlauben würde, den Umfang seines Einflusses zu übersehen, und wo, wann und weshalb er wirksam war. ${ }^{1}$ Das gleiche gilt für Bakunin. ${ }^{2}$

B. Die Bibliographien eines einzelnen Werkes werden immer Ausnahmen bleiben, schon vom Ausnahme-Charakter des Werkes her, dessen besondere Wichtigkeit allein es als Gegenstand einer eigenen Bibliographie qualifiziert. Ganz gleich, ob es sich dabei um eine Bibliographie auf internationaler oder auf nationaler Ebene handelt, ihr Verfasser muss auf allen vom Gegenstand seiner Untersuchung berührten Gebieten gründliche Kenntnisse besitzen. Als Beispiel kann die kleine Bibliographie des „Testaments des Pfarrers Meslier” gelten, die den Anhang zu Dommangets Curé Meslier bildet. ${ }^{3}$ Die Darstellung Dommangets beruht auf sehr vielen gründlichen Einzelforschungen, die ihn bis in Kommunal- und Parochialarchive führten. Meslier starb 1729,

${ }^{1}$ Eine „Bibliographie chronologique de l'œuvre originale de P.-J. Proudhon" findet sich in Jacques Bourgeat, P.-J. Proudhon, pere du socialisme français, Paris 1943, S. 249-257. Sie ist nur ein Inventar der Proudhon-Titel im Katalog der Bibliothèque Nationale.

2 Es gibt die zehn Seiten, die Nettlau Bakunin gewidmet hat in seiner S. 1, Anm. 1 angeführten Bibliographie („Michel Bakounine”, a.a.O., S. 42-51), und einige kurze Listen, wie die von Ernst Drahn, „Versuch einer Bakunin-Bibliographie von Schriften, die in Deutschland und in deutscher Sprache erschienen", in: Die Aktion, Berlin 1923, Jg. XXIII, S. 299-301. Es ist zu hoffen, dass Arthur Lehning das von ihm herausgegebene Bakunin-Avchiv mit einem bibliographischen Bande abschliesst.

3 Maurice Dommanget, Le curé Meslier. Athée, communiste et vévolutionnaire sous Louis XIV , Paris, Julliard, 1965, 552-[3] S. Die „Bibliographie et iconographie” auf S. 513-549. 
und sein Text wurde nur in Abschriften heimlich verbreitet, bis Voltaire 1761 ein Fragment in Buchform drucken liess. ${ }^{1}$ Als kritischer Bibliograph hat sich Dommanget die Frage gestellt, in welchem Masse Voltaires Auszug und die erste vollständige Ausgabe des Textes (1864) dem Manuskript Mesliers getreu sind. Seine Bibliographie beginnt also logischerweise mit einer Beschreibung von neun zeitgenössischen Manuskripten oder Kopien, die Dommanget in französischen und belgischen Bibliotheken wiederaufgefunden hat. ${ }^{2}$ Erst nach einer sorgfältigen philologischen, theologischen und historischen Untersuchung ihrer Authentizität geht Dommanget zur Bibliographie der gedruckten Texte über.

Wir stehen kurz vor der Hundertjahrfeier der Erstveröffentlichung des Marx'schen Kapital und können wohl damit rechnen, dass seine Bibliographie schon vorbereitet wird. Wird diese Arbeit die erhaltenen Manuskripte berücksichtigen und Engels' Herausgebertätigkeit der Bände II und III untersuchen? Wird sie auch die wissenschaftliche Diskussion ausserhalb der Arbeiterbewegung berücksichtigen, wie z.B. die Besprechungen des Buches in Fachzeitschriften und die Analysen in den Werken anderer Verfasser? Wird sie sich über die Qualität der Uebersetzungen aussprechen? Wenn es sich um eine Kollektivarbeit handeln sollte, wie wird die Arbeit dann verteilt und wieder zusammengefasst? Welche Lehren wird man aus den bereits erschienenen vergleichbaren Arbeiten ziehen?

$\mathrm{Zu}$ den vergleichbaren Arbeiten möchten wir auch die Abhandlungen und Monographien zählen, die eine Einzelschrift oder eine Zeitschrift zum Gegenstand der Untersuchung gemacht haben. Die ihnen zugrunde liegende Forschungsarbeit ist häufig genau dieselbe, welche auch für die spezialisierte Bibliographie geleistet werden muss. So enthält z.B. Mönkes Arbeit über Engels' „Lage der arbeitenden Klasse"3 alle Elemente, die auch in einer gleichzeitig erschienenen rein bibliographischen Arbeit über dasselbe Werk vorkommen. ${ }^{4}$ Aber die von ihm gewählte Form hat erlaubt, darüber hinaus eine ganze Anzahl auf Engels' Werk bezüglicher Veröffentlichungen zu berücksichtigen, welche von der Bibliographie ausgeschlossen werden mussten, weil sie keine Fragmente oder Zitate aus der "Lage” enthalten.

1 Der Extrait des sentiments de Jean Meslier erschien Ende 1761 als Broschüre von 63 Seiten in Genf, ohne Jahrzahl und ohne Ortsangabe.

Im Amsterdamer IISG befindet sich seit 1937 ein Manuskript von 1055 Folioseiten, das Dommanget unbekannt geblieben ist.

3 Wolfgang Mönke, Das literarische Echo in Deutschland auf Friedrich Engels' Werk "Die Lage der arbeitenden Klasse in England", Berlin, Akademie, 1965, $127 \mathrm{~S}$.

4 Bert Andréas, „Marx et Engels et la gauche hégélienne”, in: Annali, 1965, Jg. VII, S. 353-526 (die Bibliographie der „Lage”: S. 405-433). 
C. Die spezialisierten Bibliographien und Repertorien, welche ein bestimmtes Thema zum Gegenstand haben, sind zahlreich und wir beschränken uns hier darauf, einige wenige zu nennen. Der Einfluss der russischen Revolution von 1905 wird in einer Arbeit untersucht, deren Verfasser diesen Einfluss an Hand von Dokumenten in den deutschen Staatsarchiven nachweisen ; ${ }^{1}$ sie zitieren zahlreiche Fragmente aus den Akten, und ihre beiden Bände haben daher eher den Charakter von Regesten. Eine „Bibliographie zur Geschichte der Novemberrevolution” von 1918 in Deutschland hat das Berliner IML hergestellt. Sie berücksichtigt nur Bücher und Broschüren.2 Nach dem guten Beispiel mehrerer Bibliographien sind hier für jeden Titel der Standort und die jeweiligen Bibliotheks-Signaturen angegeben. Bei dem zunehmenden internationalen und Fernleihverkehr erleichtern diese Angaben die Forschung besonders.

Wenn auch zwischen dem Bibliothekskatalog und der Bibliographie unterschieden werden soll, so gibt es doch Kataloge, deren Verfasser bibliographische Arbeitsmethoden haben anwenden müssen, und die für den Forscher und Bibliographen wichtige Quellen darstellen können. Dies ist z.B. der Fall des „Katalog der Bibliothek Lenins im Kreml" der für die fast 8500 Titel dieser Sammlung zahlreiche interessante Einzelheiten angibt, wie z.B. Autorenwidmungen und Lenins Randbemerkungen. ${ }^{3}$ In Berlin wird die Veröffentlichung eines derartigen Katalogs der Bibliotheken von Marx und Engels vorbereitet, eine Arbeit, die besondere Schwierigkeiten bot, weil die Bibliotheken aufgelöst worden sind und nur ein Teil der Bücher im alten SPD Archiv in Amsterdam bewahrt geblieben ist, und ihre Titel ausserdem ohne besondere Kennzeichnung im allgemeinen Katalog ${ }^{4}$ untergegangen waren, den die SPD hatte aufstellen lassen. ${ }^{5}$

Die in einem bestimmten Lande oder in einer bestimmten Sprache erschienenen Marx/Engels-Texte sind Gegenstand einer stets zu-

1 Walter Nissen und Gerhard Schrader, Die Auswirkungen der ersten russischen Revolution von 1905-1907 auf Deutschland, Berlin, Rütten \& Loening, 1954 \& 1956, 2 Bde, LXXVI-335 \& LXIII-[3]-350-[1] S.

2 [Günter Aurich,] Bibliographie zur Geschichte der Novembervevolution, Berlin 1959, in-4, [3]-292-[1]-70 S. Polykopie. Dieser Band enthält 2351 Titel; ein zweiter Band, der die Zeitungs- und Zeitschriften-Literatur enthalten sollte, ist offenbar nicht erschienen.

${ }^{8}$ Biblioteka V.I. Lenina v Kremle. Katalog, Moskau IML, 1961, 763-[1] S.

4 Bibliothek der Sozialdemokratischen Partei Deutschlands, Systematischer Katalog, 3 Bde und 1 Nachtrag, Berlin 1927 \& 1930 (Polykopie). Ein Exemplar dieses Kataloges, sowie das Manuskript eines weiteren Nachtrags (Dezember 1927 bis März 1933) im IISG, Amsterdam.

${ }^{5}$ Die angekündigte Arbeit wird daher, im Gegensatz zum Katalog der LeninBibliothek, bestenfalls eine teilweise Rekonstruktion bieten können. 
nehmenden Zahl von Bibliographien. ${ }^{1}$ Die Gliederung und der Wert dieser Arbeiten, mitunter auch ihre Zielsetzung, sind sehr unterschiedlich. Die meisten Verfasser gehen von der Einheit des Marx/Engels' schen Werkes aus, stellen es aber, bis auf wenige Ausnahmen, in drei verschiedenen Chronologien dar, deren erste die Marx'schen, die zweite die Engels'schen und die dritte die gemeinsamen Texte enthalten. Die ukrainische Bibliographie enthält noch eine vierte Chronologie für die in der Ukraine erschienenen russischen Uebersetzungen. ${ }^{2}$ In dem Bestreben, die Ursprünge des Marxismus in ihrem Lande festzustellen, beginnen die Bibliographen der sozialistischen Länder die Chronologie jeweils mit dem Jahre, in welchem die erste Uebersetzung eines Textes erschien, ohne sein Entstehungsdatum zu berücksichtigen. ${ }^{3}$ Eine ganze Anzahl dieser Arbeiten bringen Einzelheiten über die Uebersetzer, die Auflagenhöhe usw., und die meisten repertorieren im Anhang die übersetzten Briefe von Marx und Engels. Die italienische Marx-Engels Bibliographie, ${ }^{4}$ eine der ausführlichsten, hat nur eine fortlaufende Chronologie, welche die Schriften beider Autoren nach dem Entstehungsdatum anführt, das für jeden Text genau angegeben ist. Der Originaltitel sowie Ort und Jahr der Erstveröffentlichung gehen jeweils der wiederum chronologischen Liste der italienischen Uebersetzungen voraus. Ein besonderer Index für die Periode von 1848 bis 1889 erlaubt jedoch, die Reihenfolge der in diesen Jahren veröffentlichten Texte

1 Eine Bibliographie dieser Bibliographien ist zusammengestellt worden von L. A. Levin, Bibliografija bibliografij proizvedenij K. Marksa, F. Engel'sa, V. I. Lenina, Moskau 1961, 238-[1] S. Von den insgesamt angeführten 886 Titeln betreffen 383 Marx und Engels. Hiervon erschienen

von 1872 bis 1917: 10 Titel mit 105 Seiten;

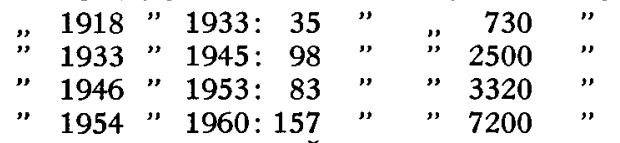

${ }^{2}$ I. A. Bagmut und P. M. Smorgun, Vidannja tvori K. Marksa $i$ F. Engel'sa na Ukrajni, Kharkov 1962, 74-[2] S. Führt 274 Titel an.

- Marks i Engels w Polsce. Materiaty do bibliografii 1842-1952, Warschau 1953, 111-[1] S.; Miklós Práger, Marx és Engels müveinek magyar bibliográfiája, Budapest 1953, $80 \mathrm{~S}$. (vom selben Verfasser ein polykopiertes Supplement, für den internen Gebrauch der ungarischen Institute bestimmt: Pótkötet. Az 1953. évi kiadás hiányainak pótlása, kiegészitve az 1953-1963. években megjelent müvekkel, Budapest 1964, 141 S.); George Baiculescu, Marx si Engels in limba romina 1871-1944, Bukarest 1956, 60-[3] S.; T. Borov, Karl Marks i Fridrikh Engels na bolgarski. Bibliografija, Sofia 1961, 198-[2] S. Der tschechischen Bibliographie ist in erster Linie daran gelegen, die greifbaren modernen Ausgaben nachzuweisen, und die jüngsten Ausgaben erscheinen daher an erster Stelle: Miloš Papírnfk, Bibliografický prüvodce ke klasikum marxismu-leninismu, Prag 1956, 130-[2] S. (eine zweite ergänzte Auflage, Prag 1965, 128-[3] S.).

- Gian Mario Bravo, Marx e Engels in lingua italiana 1848-1960, Mailand, Avanti!, 1962, 175-[2] S. 
festzustellen. Die allgemeine Wichtigkeit dieser nationalen Bibliographien besteht u.a., wie Rubel und Blumenberg schon zu Recht hervorgehoben haben, darin, dass sie als vorbereitende Arbeiten einen unentbehrlichen Beitrag zur Herstellung der integralen Bibliographie der Werke Marx/Engels leisten.

\section{III}

Wir haben in dieser fragmentarischen und häufig vielleicht zu kritischen Uebersicht über den heutigen Stand der Bibliographie der Arbeiterbewegung gewiss mehr Fragen gestellt als Lösungen vorgeschlagen. Sie erlaubt aber vielleicht doch, die Richtung zu zeigen, in der künftige Lösungen gesucht werden können.

Die geschichtswissenschaftlichen Methoden sind in unserer Zeit in vielen Hinsichten vervollkommnet worden. Diese Entwicklung stellt ihrerseits erhöhte Anforderungen an die Hilfswissenschaften. Auf dem Gebiete der Bibliographie der allgemeinen Arbeiterbewegung gehört z.B. die Zeit, welcher Stammhammers Inventar noch genügen konnte, endgültig der Vergangenheit an.

Doch arbeiten auch heute noch viele Bibliographen, wie zu Stammhammers Zeiten, zu isoliert. Die Koordination der laufenden bibliographischen Arbeiten und gegenseitige Information sind Immediatforderungen. Die bisherigen sporadischen und individuellen Kontakte müssen in regelmässige organisierte Formen gebracht werden. Ein erster Schritt wäre die periodische Veröffentlichung, auf internationaler Ebene, von Mitteilungen über Arbeitsvorhaben, laufende Arbeiten und verteidigte aber unveröffentlichte Dissertationen. Dieser erste Vorschlag scheint uns ohne allzu grosse Schwierigkeiten durchführbar zu sein. ${ }^{1}$ Anders ist es mit einem zweiten bestellt, den wir hier nur in Erwägung geben möchten, ohne auf Einzelheiten einzugehen. Für jegliche bibliographische Forschungsarbeit wäre das Vorhandensein einer Bibliographie der Bibliographien unseres Gebietes von grosser

1 Ueber laufende Arbeiten und Vorhaben in England und in der Bundesrepublik Deutschland unterrichten bereits das Bulletin of the Society for the Study of Labour History, London 1965, Nr. 11 (S. 66-74 „Research in Progress”) und die Internationale wissenschaftliche Korrespondenz zur Geschichte der deutschen Arbeiterbewegung, Berlin, Nr. 1 (2. verb. Ausgabe Mai 1966, S.33-52: Henryk Skrzypczak, „Forschungs- und Publikationsvorhaben zur Geschichte der deutschen Arbeiterbewegung und angrenzender Gebiete"). Dissertationen über Arbeiterbewegung und Sozialismus sind nachgewiesen in Annali, 1960, Jg. III, für Oesterreich (S. 827-837, von Karl Selber), Australien (S. 838-841, von Henry Mayer) und Neuseeland (S. 842-845, von Herbert Roth). Die vom Berliner IML herausgegebenen Beiträge zur Geschichte der deutschen Arbeiterbewegung bringen seit einigen Jahren regelmässig „Berichte über verteidigte Dissertationen”. 
Bedeutung. Ihre Herstellung wird eine sorgfältige Vorbereitung und vor allem internationale Zusammenarbeit erfordern.

Im Zusammenhang mit den heutigen erhöhten Anforderungen der Geschichtswissenschaften haben wir mehrfach den Ausdruck „Bibliograph und Forscher" benutzt. Nur dieser neue Typus des Bibliographen wird nach unserer Ansicht imstande sein, die neuen Aufgaben zu lösen. Er kann natürlich nicht fix und fertig erfunden werden. Wir alle werden unsere Methoden zu vervollkommnen, unsere Kenntnisse zu bereichern und gleichzeitig für den Nachwuchs zu sorgen haben, uns um die Ausbildung der künftigen Bibliographen kümmern müssen. Hierzu schlagen wir das Organisieren periodisch abzuhaltender internationaler Seminare vor, an denen erfahrene Bibliographen und junge Forscher teilnehmen. Sie würden die gegenwärtigen und neuen Methoden, Quellenprobleme usw. gründlich diskutieren und dabei die Probleme der Herstellung von Bibliographien unter dem Gesichtspunkt ihrer Benutzung betrachten und sie als „Erkenntnisinstrument historischer und philologischer Zusammenhänge" auffassen müssen, wie das kürzlich ein Verfasser ausgedrückt hat. ${ }^{1}$ Sehr erwünscht wäre deshalb die Beteiligung von Historikern, die den Herstellern ihrer Arbeitsinstrumente selbst erklären könnten, was sie von ihnen erwarten. Derart könnte der Historiker vom einfachen Benutzer zum tätigen Mithersteller werden, der mit dem Bibliographen, z.B. in einer Arbeitsgruppe, auch Spezialarbeiten vorbereiten könnte, wie die Handbücher, welche der Universitätsunterricht zur Einführung in die Forschung verlangt.

1 Wolfgang Mönke, „Andréasa Bibliografia Manifestu Komunistycznego”, in: Studia filozoficzne kwartalnik, Warszawa, 1966, Heft 1, S. 222. 